\title{
Vesicle-related microRNAs in plasma of nonsmall cell lung cancer patients and correlation with survival
}

\author{
J. Silva*,`, V. García*,§ Á. Zaballos ${ }^{\#, ~ M . ~ P r o v e n c i o *, ~ L . ~ L o m b a r d i ́ a ", ~}$ \\ L. Almonacid ${ }^{\#}$, J.M. García*, G. Domínguez*, C. Peña*, R. Diaz*, \\ M. Herrera*, A. Varela ${ }^{+}$and F. Bonilla*
}

ABSTRACT: The identification of tumour biomarkers that detect the presence of disease using noninvasive diagnostic procedures is a key part of cancer research. We determined in plasma the vesicle-related microRNA (miRNA) expression profile of nonsmall cell lung cancer (NSCLC) and evaluate whether plasma miRNAs can be both discriminating (between patients and healthy controls) and prognostic markers.

365 human miRNAs were analysed by Taqman $®$ low-density arrays (Applied Biosystems, Foster City, CA, USA) in the plasma from 28 NSCLC patients and 20 controls. Five selected miRNAs (let7f, miR-20b, miR-30e-3p, miR-223 and miR-301) were validated independently by real-time PCR in plasma from 78 NSCLC and 48 controls and correlated with pathologic parameters and survival.

Levels of let-7f, miR-20b and miR-30e-3p were decreased in plasma vesicles of NSCLC patients. Moreover, levels of let-7f and miR-30e-3p distinguished between two groups of patients for stage of disease and therefore possibility of surgery. Plasma levels of miR-30e-3p and let-7f were associated with short disease-free survival and overall survival, respectively.

NSCLC patients and healthy controls differ in vesicle-related miRNAs in plasma. Levels of let-7f and miR-30e-3p in NSCLC patients are associated with poor outcome. Thus, plasma vesiclerelated miRNAs obtained by noninvasive methods could serve as circulating tumour biomarkers of discriminating and prognostic value.

KEYWORDS: MicroRNAs, microvesicles, nonsmall cell lung cancer, plasma

$\mathbf{T}$ he identification of tumour biomarkers to detect the presence of disease using noninvasive diagnostic procedures is a key part of cancer research. This research is mainly important in lung cancer, for which imaging or cytological techniques have not significantly affected overall mortality [1]. Several studies have demonstrated the presence of extracellular nucleic acids in cancer patients as well as their potential as surrogate sources of tumour nucleic acids [2, 3]. This establishes the noninvasive clinical potential and prognostic value of the analysis of plasma nucleic acids in cancer patients as tumour biomarkers.

Regarding the release mechanism of nucleic acids from the tumour cells to the bloodstream, we recently reported that a major fraction of these molecules detected in plasma of cancer patients are highly protected in tumour specific microvesicle-like structures [4]. As release of microvesicles provides an intercellular communication mechanism, it seems clear that the cell type from which they were derived and their composition will determine their function. Initially characterised as a mechanism to remove obsolete proteins [5], several studies support a role in tumour immunity, since microparticles present tumour antigens to sensitised T-cells or dendritic cells and promote tumour rejection [6-8]. In contrast, these vesicles have also been involved in immune suppression, since they could transport molecules responsible for the detrimental effects exerted by tumour cells on the immune system [9-11]. Recently, we and others reported that these microparticles are enriched in mRNAs and microRNAs (miRNAs) [4, 12-14]. Moreover, the RNA contained in vesicles from donor mouse mast cells was transferable to recipient human
AFFILIATIONS

Depts of *Medical Oncology, and +Thoracic Surgery, University Hospital Puerta de Hierro Majadahonda,

${ }^{\#}$ Genomics Functional Unit, Dept of Immunology and Oncology, Centro Nacional de Biotecnología, and, - Spanish National Cancer Research Centre (CNIO), Madrid, Spain.

${ }^{\S}$ These authors contributed equally to this study.

CORRESPONDENCE

F. Bonilla

Dept of Medical Oncology

Puerta de Hierro Majadahonda

University Hospital

C/Joaquín Rodrigo

2; E-28222 Majadahonda

Madrid

Spain

E-mail: fbonilla.hpth@

salud.madrid.org

Received:

Feb 232010

Accepted after revision:

June 072010

First published online:

July 012010 
mast cells, which was then translated, which indicated that the transferred RNA was biologically active [12]. Similarly, previous studies suggested that miRNAs contained in tumour vesicles are functional and suppress the target mRNAs for signal transduction components within T-cells [9].

As master regulators of gene expression, abnormal levels of miRNAs in tumours have important pathogenetic consequences. miRNAs act as tumour suppressors when they repress oncogenic genes, or as oncogenes when they downregulate tumour suppressors [15]. Furthermore, analyses of several human cancers have identified miRNA signatures relating to initiation, progression, diagnosis or prognosis of tumours [16]. Cancer-specific miRNAs with high resistance to RNases and harsh conditions have been identified in plasma or serum of cancer patients [1,17-21] evidencing that this high stability conferred by its particle-associated features makes miRNA levels well suited for being tested as cancer biomarkers in patient plasma samples.

In our study, we performed a Taqman ${ }^{\circledR}$ (Applied Biosystems, Foster City, CA, USA) quantitative PCR analysis of vesiclerelated miRNAs from plasma of NSCLC patients. This approach identified five miRNAs (let-7f, miR-20b, miR-30e$3 p, \operatorname{miR}-223$ and miR-301), whose levels distinguish people with tumours from the healthy population. Quantitative analysis of these miRNAs in a large series of NSCLC patients showed that several of them were potentially useful for diagnosis and prognosis of the tumours.

\section{METHODS}

\section{Plasma samples and patient characteristics}

We used a final series of 106 NSCLC patients (March, 2004 to April, 2007), 28 included in the testing series and 78 in the validation set, who were followed until April 2009. Informed written consent was obtained from all participants after an explanation of the nature of the study, as approved by the research ethics board of our hospital (University Hospital Puerta de Hierro Majadahonda, Madrid, Spain). Blood samples were taken either before intervention on the day of surgery or before chemotherapy in non-operable patients. Blood samples from a final series 68 healthy donors, 20 included in the testing series and 48 in the validation set, were obtained at the haematology unit of our hospital. Plasma was prepared by serial centrifugations [4]. The diagnosis of NSCLC in all tumour specimens was confirmed histologically. Clinicopathological parameters, clinical follow-up and treatment from all patients are described in the supplementary material.

\section{Vesicles and miRNA isolation}

$3 \mathrm{~mL}$ of plasma were incubated for $30 \mathrm{~min}$ with $100 \mu \mathrm{L}$ of super-paramagnetic beads coated with epithelial antibody BerEP4 in line with Dynabeads Epithelial Enrich protocol (Dynal Biotech ASA, Oslo, Norway). miRNAs were extracted from the Dynabeads fraction by magnetic separation with the mirVana $^{\mathrm{TM}}$ miRNA Isolation Kit (Ambion Inc, Austin, TX, USA) in line with the manufacturer's instructions. cDNA was synthesised from vesicle-related miRNA by use of genespecific primer pools, following the TaqMan MicroRNA Assay protocol (Applied Biosystems).

\section{miRNAs Taqman ${ }_{\circledast}$ low-density arrays}

Real-time PCR of 365 human miRNAs was performed with Taqman ${ }$ low-density arrays (TLDA; Applied Biosystems; TaqMan ${ }$ miRNA assays human panel early access kit). The Ct data were obtained by the SDS v2.2 software with automatic threshold settings.

To find suitable normaliser miRNAs [22], GENORM software was used with the $\mathrm{Ct}$ values of the 50 most expressed miRNAs in the whole sample set. miRNAs miR-142-3p and miR-30b were found to be the most stable miRNAs and geometric normalisation applied to $\mathrm{Ct}$ values. Normalised data were processed with the Preprocessor Tool [23] included in the GEPAS package [24]. Differentially expressed miRNAs were identified by using significance analysis of microarrays (SAM) [25] and ANOVA with the linear models method [26]. More information about TLDA, data processing and analysis are included in the supplementary material.

\section{Real-time PCR for independent series validation}

Plasma miRNA levels of let-7f, miR-20b, miR-30e-3p, miR-223 and miR-301 were normalised using the geometric average [22] of the two most stable miRNAs detected in miRNA TLDA, miR$142-3 p$ and miR-30b. Relative concentrations of the miRNAs were calculated using a standard curve of each one plotted from serial dilution of cDNA from tumour tissues. Quantitative PCR was performed with the TaqMan ${ }_{\circledast}$ MicroRNA Assay specific for each miRNA (Applied Biosystems), in line with the manufacturer's instructions.

\section{Statistical analysis}

Plasma miRNA levels were not normally distributed (determined by the Kolmogorov-Smirnov test), so the significance of plasma miRNA levels was determined by the Mann-Whitney test. Box-plots were performed with a $\log _{10}$ transformation of data. The relationship between the cumulative probability of overall survival (OS) and disease-free survival (DFS), as well as analysed predictors, was calculated by the Kaplan-Meier method, while significant differences between curves were evaluated with Mantel's log-rank test. A p-value $<0.05$ was considered statistically significant. Statistical analysis was performed by the SPSS software (version 13.0; SPSS Inc., Chicago, IL, USA).

\section{RESULTS}

Profiling of plasma samples with microRNA Taqman ${ }_{\mathbb{R}}$ arrays Previous to array running, plasma RNA samples were probed with Taqman ${ }_{\circledR}$ assays for let-7a and miR-16 miRNAs and those giving $\mathrm{Ct}$ values $>30$ cycles were discarded for the array assay. Finally, 20 plasma RNA samples from normal subjects and 28 from NSCLC patients were subjected to qRT-PCR in Taqman ${ }^{\circledR}$ arrays. The two populations did not differ in terms of age, sex or smoking history. Equivalent volumes of plasma RNA were then used for reverse transcription and TaqMan reactions. No differences were found in the number of miRNAs detected or in those with $\mathrm{Ct}<35$ between normal and tumour samples (mean 107 versus 102 and 81 versus 77, respectively), 30\% of the total miRNAs analysed. Of these, miR-223 consistently showed the lowest $\mathrm{Ct}$ in every sample, which is consistent with results reported elsewhere [18]. Although a different technical approach was used, other miRNAs that were detected, such as 
miR-24, miR-26a and miR-16, also showed low Ct numbers in our assays.

miRNAs expressed differently in normal and diseased conditions were identified by running SAM and ANOVA linear model methods. Table 1 shows the 10 differentially expressed miRNAs in plasma from NSCLC patients that tacking account a q-value $>3 \%$ in the SAM analysis and FDR $>50 \%$ reached the best FDR value in both analyses. We quantified by qRTPCR the expression of these miRNAs in the initial series of 20 healthy controls and 28 NSCLC patients. Statistically differences were not found between healthy and tumour populations probably due to the small number of cases in the series. Five of these (let-7f, miR-20b, miR-30e-3p, miR-223 and miR301) were randomly selected for validation with independent sample series.

\section{Validation of miRNAs}

To test the consistency of selected miRNAs, we analysed their levels by quantitative RT-PCR on a set of 78 new NSCLC plasma samples and 48 healthy donor samples, different from the ones that were part of the miRNA profiling study. No significant differences were observed for miRNAs used for internal normalisation (miR-142-3p and miR-30b) between control and NSCLC samples $(p=0.19$ and $p=0.85$, respectively; Mann-Whitney test).

We verified the decreased levels in plasma from NSCLC patients of 3 of 5 miRNAs selected. Thus, for let-7f, miR-20b and miR-30e$3 p$, there were statistically significant differences between the two populations analysed $(p=0.001, p=0.058$ and $p=0.041$, respectively; Mann-Whitney test; fig. 1). miR-223 and miR-301, which did not distinguish healthy and patient populations in this external series, were not chosen in further studies.

In addition, NSCLC patients were divided into two groups, depending on the stage of disease and therefore possibility of surgery: patients with resection of the tumours (stages I, II and IIIA) and patients without resection (stages IIIB and IV). Our data showed that let-7f and miR-30e-3p levels distinguished the two groups of patients $(p=0.05$ and $p=0.005$, respectively; fig. 2).

\section{miRNA plasma levels in NSCLC patients and outcome}

To determine whether the miRNA levels validated in the independent series could serve as prognostic markers, indicating DFS and OS, we reviewed follow-up care for the 78 NSCLC patients. The median (range) follow-up was 22 (1-59) months. During the period of follow-up, $32 \%$ of operable patients relapsed and $78 \%$ of all patients, operable and non-operable, died. For this survival analysis, NSCLC patients were grouped according to miRNA levels in bicentiles (low and high levels) or in tertiles (low, medium and high levels).

\section{DFS}

At 59 months of follow-up, DFS of the patients with resection of the tumour was 33\% (95\% CI, 14-52\%). When these patients were divided into bicentiles, a statistically significant difference was observed in DFS regarding miR-30e-3p plasma levels: the patients with low levels had a 50-month DFS rate of $13 \%$ (95\% CI, 0-35\%) and the group with high levels had a rate of $50 \%$ (95\% CI, 23-77\%; $p=0.009$; Kaplan-Meier test; fig. 3a). Levels of let-7f and miR-20b in plasma were not associated with DFS.

\section{OS}

At 59 months of follow-up, OS for patients was 19\% (95\% CI, 9-29\%). When patients were divided into tertiles, a trend to significant association was found in OS for let-7f plasma levels $(\mathrm{p}=0.077$, Kaplan-Meier test). Our data indicate that patients with low or median levels of let-7f in plasma presented similar behaviour compared with patients with high let-7f levels. Thus, the patients with low or median levels of let-7f were grouped into the same group and only two categories were analysed: patients with low or high levels. The maximum follow-up for patients with high let-7f levels was 46 months. Thus, there was statistical significance, since patients with low let-7f levels had at 46 months an OS rate of 30\% (95\% CI, 17$43 \%)$ and patients with high levels had an $8 \%$ rate $(95 \% \mathrm{CI}$,

TABLE 1 MicroRNAs differentially expressed in nonsmall cell lung cancer patients with respect to healthy controls, showing the lowest percentage of false positives

\begin{tabular}{|c|c|c|c|c|}
\hline \multirow[t]{2}{*}{ miRNA } & \multirow{2}{*}{$\begin{array}{c}\text { Mean fold change (patients } \\
\text { versus controls) }\end{array}$} & \multicolumn{2}{|c|}{ ANOVA } & \multirow{2}{*}{$\begin{array}{c}\text { SAM analysis } \\
\text { q-value (\%) }\end{array}$} \\
\hline & & Unadjusted p-value & False-discovery rate & \\
\hline hsa-let-7d & 0.717536342 & 0.0227134 & 0.2336232 & 2.48 \\
\hline hsa-miR-223 & 0.724024846 & 0.0152853 & 0.2336232 & 2.89 \\
\hline hsa-miR-383 & 0.879514588 & 0.0199543 & 0.2336232 & 2.89 \\
\hline hsa-miR-192 & 0.660891669 & 0.0579683 & 0.3794289 & 2.89 \\
\hline hsa-miR-30e-3p & 0.842891622 & 0.0674752 & 0.4048513 & 2.89 \\
\hline hsa-miR-301 & 0.719256668 & 0.1026840 & 0.4496109 & 2.89 \\
\hline hsa-let-7f & 0.537065355 & 0.1436084 & 0.4584876 & 2.48 \\
\hline hsa-miR-572 & 0.524393644 & 0.1568791 & 0.4584876 & 2.48 \\
\hline hsa-miR-20b & 0.698194300 & 0.1651005 & 0.4584876 & 2.89 \\
\hline hsa-miR-345 & 0.655958424 & 0.1678559 & 0.4584876 & 2.89 \\
\hline
\end{tabular}

SAM: significance analysis of microarrays. 

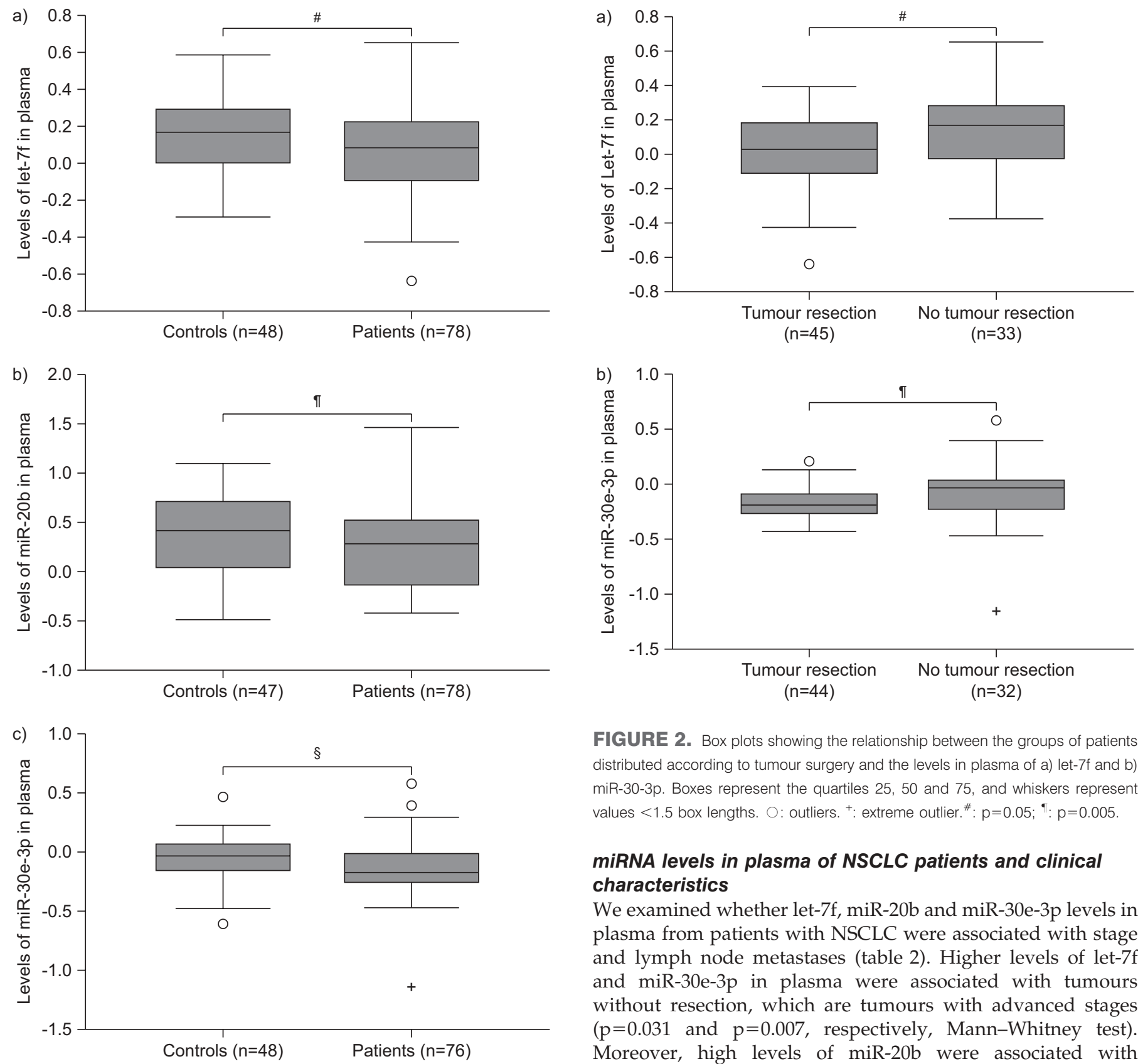

FIGURE 1. Box plots showing the relationship between the two populations studied and the levels in plasma of a) let-7f, b) miR-20b and c) miR-30e-3p. Boxes represent the quartiles 25,50 and 75 , and whiskers represent values $<1.5$ box lengths. $\bigcirc$ : outliers. ${ }^{+}$: extreme outlier. ${ }^{*}: p=0.001 ;{ }^{\uparrow}: 0.058 ;{ }^{\text {s }} 0.041$.

$0-22 \% ; p=0.038$, Kaplan-Meier test; fig. 3b). Levels of miR-30e$3 \mathrm{p}$ and $\mathrm{miR}-20 \mathrm{~b}$ in plasma were not associated with OS.

Patients without resection of the tumour have worse outcome than patients with resection of the tumour, which was also observed in our series. The maximum follow-up of patients without resection of tumour was 33 months, showing an OS rate of $3 \%(95 \% \mathrm{CI}, 0-9 \%)$, whereas patients with resection had a rate of $56 \%$ (95\% CI, $40-72 \%$; $<<0.001$, Kaplan-Meier test). For this reason, OS was analysed for each miRNA in patients distributed according to surgery treatment but no differences were found.

FIGURE 2. Box plots showing the relationship between the groups of patients distributed according to tumour surgery and the levels in plasma of a) let-7f and b) miR-30-3p. Boxes represent the quartiles 25, 50 and 75, and whiskers represent values $<1.5$ box lengths. $\bigcirc$ : outliers. ${ }^{+}$: extreme outlier. ${ }^{\#}: p=0.05 ;{ }^{\bullet}: p=0.005$.

\section{miRNA levels in plasma of NSCLC patients and clinical characteristics}

We examined whether let-7f, miR-20b and miR-30e-3p levels in plasma from patients with NSCLC were associated with stage and lymph node metastases (table 2). Higher levels of let-7f and miR-30e-3p in plasma were associated with tumours without resection, which are tumours with advanced stages $(\mathrm{p}=0.031$ and $\mathrm{p}=0.007$, respectively, Mann-Whitney test). Moreover, high levels of miR-20b were associated with advanced stages and presence of metastases in lymph nodes $(\mathrm{p}=0.008$ and $\mathrm{p}=0.01$, respectively, Mann-Whitney test).

\section{DISCUSSION}

A major area of research in the field of cancer is the identification of biomarkers that detect the presence of a tumour with noninvasive diagnostic procedures. The simplicity of getting a blood sample from a cancer patient and the existence of extracellular nucleic acids in plasma suggest that biomarkers found in blood plasma would be particularly helpful as strategies for cancer detection.

miRNAs play important roles in oncogenesis and several studies based on profiling methods in different tumour types have shown the potential of miRNAs in cancer diagnosis and prognosis [16]. Profiling of miRNA expression in tissues discriminates diseased from normal subjects and several 

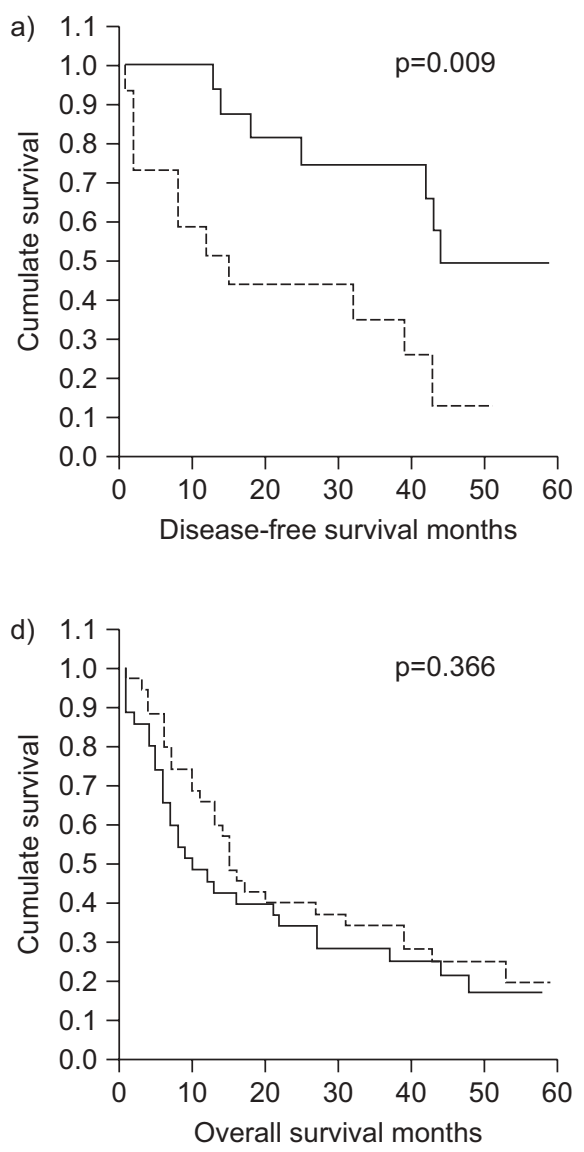

b)

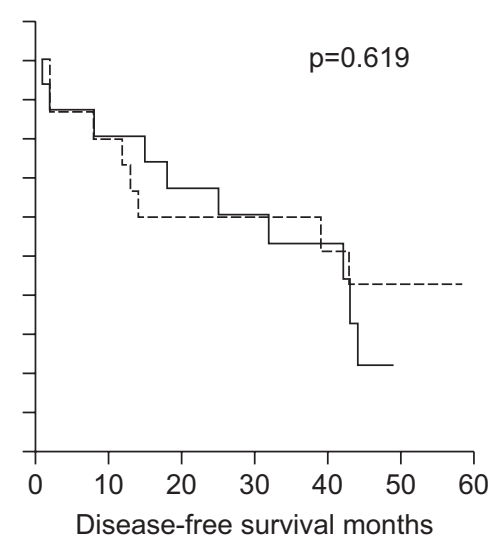

e)

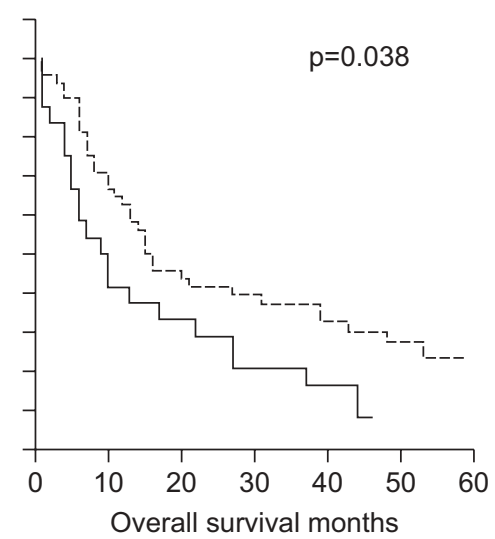

c)

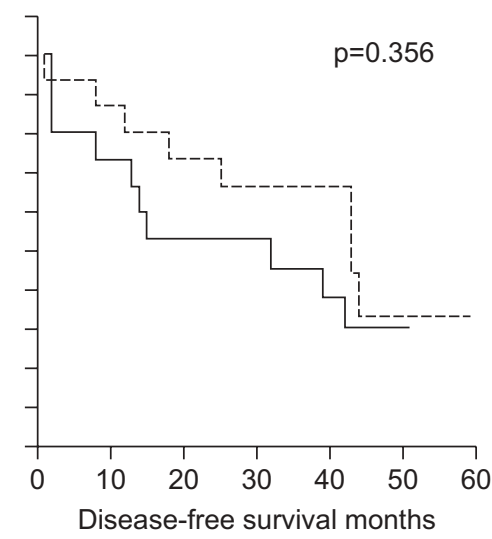

f)

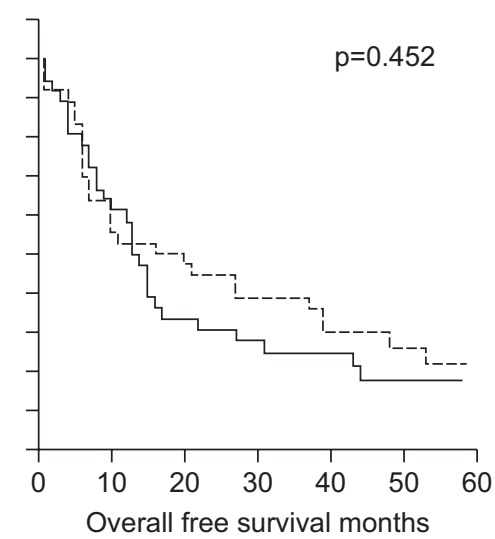

FIGURE 3. Kaplan-Meier survival curves in relation to levels of a) miR-30e-3p, b) let-7f and c) miR-20b in plasma in disease-free survival and d) miR-30e-3p, e) let-7f and f) miR-20b in plasma in overall survival. —_: high levels; -----: low levels.

miRNAs have been proposed as potential biomarkers in cancer studies [27]. Furthermore, tumour cells release microparticles enriched in functional miRNAs to the blood [4, 12-14]. We consider that both the high stability conferred by the particles' characteristics and the involvement of miRNAs in cancer

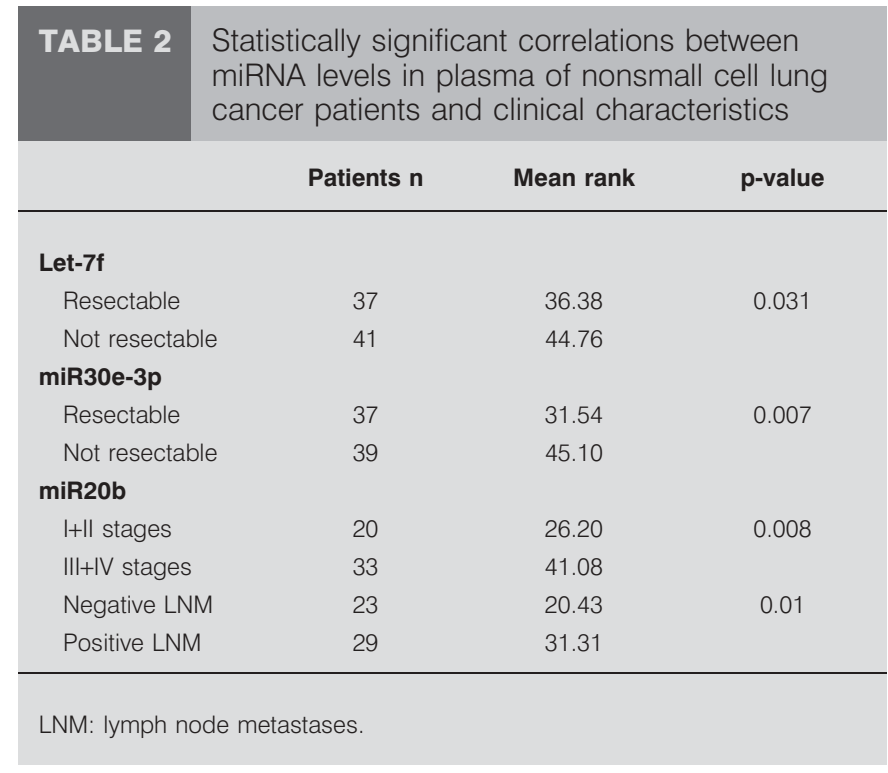

oncogenesis support the idea of testing miRNA levels in plasma of cancer patients as a possible cancer biomarker.

To determine whether miRNAs enter the blood-stream at levels that can be measured and used as cancer biomarkers, Taqman $_{\mathbb{R}}$ stem-loop qRT-PCR was applied. qRT-PCR is a reliable and affordable method for miRNA profiling, as methods for high throughput have been developed and, in contrast to microarray-based studies, quantitative data are obtained in a single processing experiment. TaqMan $®$ miRNA arrays enable analysis of 365 human miRNAs on a microfluidic card with minimal sample processing. In our study, comparison of specific profiles of miRNAs contained in microvesicles from plasma of healthy controls and plasma of NSCLC patients revealed that decreased levels of several miRNAs discriminated nontumour from tumour population. It is difficult to reconcile the observation that specific tumour exosome-secreted miRNAs are lower in the blood of cancer patients compared with healthy controls; however, validation of levels of these miRNAs by real-time quantification in an external series confirmed that levels of let-7f, miR-20b and miR-30b statistically decreased in microparticles of NSCLC patients. Moreover, our results supported previous data by others who found that the copy number of these three miRNAs decreased in lung cancer serum more than in normal serum [19]. Furthermore, our data showed that levels of these plasma miRNAs distinguished the two groups of patients for stage of 
disease and therefore possibility of surgery. Thus, levels of let$7 \mathrm{f}$ and miR-30e differentiated populations of patients with resectable tumours and patients with nonresectable tumours.

The let-7 family contains a dozen of the most actively studied tumour suppressor miRNAs [28]. Their reduced expression has been described in human lung cancer and correlates with poor prognosis $[29,30]$. Moreover, the chromosomal location of various let-7 genes is frequently deleted in a variety of human cancers and several of them can be directly repressed by the c-Myc oncoprotein [31]. Interestingly, in our series of NSCLC patients, the higher let-7f levels were detected in the group of patients with nonresectable or more malignant tumours. In addition, OS was shorter in this population. Thus, we suggested a possible association between let-7f and poor prognosis of NSCLC. The apparent discrepancy observed between decreased levels of let-7f in NSCLC patients compared with healthy controls and the fact that increased levels of this miRNA were associated with worse prognosis may be explained by several causes. First, let7-f has been previously identified as a pro-angiogenic miRNA [32]. Thus, in this sense, it is not unexpected to observe an association between levels of circulating pro-angiogenic let-7f and tumours with worse prognosis. Alternatively, there are two variants of this miRNA, let-7f1 and let-7f2, with nonreported functions and with different chromosomal locations, different sizes but with high sequence homology. The Taqman ${ }$ miRNA used in quantification of let-7f levels links to both variants; therefore, it was not achievable to discriminate between them. An unresolved possibility is that each variant has diverse roles in tumourigenesis and that the abundance of each variant shifts in plasma from healthy and in cancer population.

The oncogenic potential of miR-20b has been suggested in previous studies in several tumour types. In human carcinomas, accumulation of miR-20b is involved in human T-cell leukaemia [33] and high expression is associated with lower probability of survival in gastric cancer patients [34]. Our data support this oncogenic role of miR-20b, since patients with more advanced stage of disease and presence of lymph node metastases had higher microvesicle-related miR-20b levels.

miR-30e, a member of the miR-30 family, is a negative regulator of Ubc9 expression. Ubc9 plays a critical role in sumoylation-mediated cell pathways involving cell growth and cancer development. Recently, it was reported that, whereas Ubc9 was upregulated in several tumour types, such as breast, head and neck and lung carcinomas, miR-30e was under-expressed. Moreover, ectopic expression of miR-30e suppressed cell growth $[35,36]$. In our study of clinical outcome, Kaplan-Meier analysis showed that NSCLC patients with lower levels in plasma vesicles of miR-30e had shorter disease-free survival.

Thus, we established that tumour-derived miRNAs detected in microparticles present in plasma from NSCLC patients can serve as circulating tumour biomarkers for detection of a major human cancer type. We suggest that plasma miRNA-based qPCR assays could distinguish NSCLC in a noninvasive diagnostic procedure. This purpose is supported by a recent study that has identified a miRNA signature in genome-wide serum miRNA expression profiling that predicted outcome surveillance in shorter-survival and longer-survival groups of NSCLC patients. Further, to our knowledge, this is the first report on quantitative assessment of plasma microvesicular miRNAs in NSCLC patients to put forward a screening test for NSCLC that affects overall mortality and survival. The associations described here between levels of let-7f, miR-20b and miR-30e and poor prognosis parameters and survival supported the role of these miRNAs as biomarkers for NSCLC.

\section{STATEMENT OF INTEREST}

None declared.

\section{ACKNOWLEDGEMENTS}

We are grateful to M. Eaude (University of Barcelona, Barcelona, Spain) for correcting the English.

\section{REFERENCES}

1 Rabinowits G, Gerçel-Taylor C, Day JM, et al. Exosomal microRNA: a diagnostic marker for lung cancer. Clin Lung Cancer 2009; 10: 42-46.

2 Mandel P, Métais P. Les acides nucléiques du plasma sanguin chez l'Homme. CR Acad Sci Paris 1948; 142: 241-243.

3 Johnson PJ, Lo YM. Plasma nucleic acids in the diagnosis and management of malignant disease. Clin Chem 2002; 48: 1186-1193.

4 García JM, García V, Peña C, et al. Extracellular plasma RNA from colon cancer patients is confined in a vesicle-like structure and is mRNA-enriched. RNA 2008; 14: 1424-1432.

5 Johnstone RM, Adam M, Hammond JR, et al. Vesicle formation during reticulocyte maturation. Association of plasma membrane activities with released vesicles (exosomes). J Biol Chem 1987; 262: 9412-9420.

6 Zitvogel L, Regnault A, Lozier A, et al. Eradication of established murine tumours using a novel cell-free vaccine: dendritic cellderived exosomes. Nat Med 1998; 4: 594-600.

7 Wolfers J, Lozier A, Raposo G, et al. Tumour-derived exosomes are a source of shared tumour rejection antigens for CTL crosspriming. Nat Med 2001; 7: 297-303.

8 Chen W, Wang J, Shao C, et al. Efficient induction of antitumour T cell immunity by exosomes derived from heat-shocked lymphoma cells. Eur J Immunol 2006; 36: 1598-1607.

9 Taylor DD, Gerçel-Taylor C. Tumour-derived exosomes and their role in cancer-associated T-cell signalling defects. $\mathrm{Br} J$ Cancer 2005; 92: 305-311.

10 Whiteside TL. Tumour-derived exosomes or microvesicles: another mechanism of tumour escape from the host immune system? Br J Cancer 2005; 92: 209-211.

11 van Niel G, Porto-Carreiro I, Simoes S, et al. Exosomes: a common pathway for a specialized function. J Biochem 2006; 140: 13-21.

12 Valadi H, Ekström K, Bossios A, et al. Exosome-mediated transfer of mRNAs and microRNAs is a novel mechanism of genetic exchange between cells. Nat Cell Biol 2007; 9: 654-659.

13 Taylor DD, Gercel-Taylor C. MicroRNA signatures of tumourderived exosomes as diagnostic biomarkers of ovarian cancer. Gynecol Oncol 2008; 110: 13-21.

14 Hunter MP, Ismail N, Zhang $X$, et al. Detection of microRNA expression in human peripheral blood microvesicles. PLoS One 2008; 3: e3694.

15 Chivukula RR, Mendell JT. Circular reasoning: microRNAs and cell-cycle control. Trends Biochem Sci 2008; 33: 474-481.

16 Cho WC. OncomiRs: the discovery and progress of microRNAs in cancers. Mol Cancer 2007; 6: 60.

17 Lawrie CH, Gal S, Dunlop HM, et al. Detection of elevated levels of tumour-associated microRNAs in serum of patients with diffuse large B-cell lymphoma. Br J Haematol 2008; 141: 672-675. 
18 Mitchell PS, Parkin RK, Kroh EM, et al. Circulating microRNAs as stable blood-based markers for cancer detection. Proc Natl Acad Sci USA 2008; 105: 10513-10518.

19 Chen X, Ba Y, Ma L, et al. Characterization of microRNAs in serum: a novel class of biomarkers for diagnosis of cancer and other diseases. Cell Res 2008; 18: 997-1006.

$20 \mathrm{Ng}$ EK, Chong WW, Jin $\mathrm{H}$, et al. Differential expression of microRNAs in plasma of colorectal cancer patients: a potential marker for colorectal cancer screening. Gut 2009; 58: 1375-1381.

21 Zhu W, Qin W, Atasoy U, et al. Circulating microRNAs in breast cancer and healthy subjects. BMC Res Notes 2009; 2: 89.

22 Vandesompele J, De Preter K, Pattyn F, et al. Accurate normalization of real-time quantitative RT-PCR data by geometric averaging of multiple internal control genes. Genome Biol 2002; 3: RESEARCH0034.

23 Herrero J, Díaz-Uriarte R, Dopazo J. Gene expression data preprocessing. Bioinformatics 2003; 19: 655-656.

24 Herrero J, Al-Shahrour F, Díaz-Uriarte R, et al. GEPAS, a webbased resource for microarray gene expression data analysis. Nucleic Acids Research 2003; 31: 3461-3467.

25 Tusher VG, Tibshirani R, Chu G. Significance analysis of microarrays applied to the ionizing radiation response. Proc Natl Acad Sci USA 2001; 24: 5116-5121.

26 Smyth GK, Ritchie M, Thorne NP, et al. Limma: Linear Models for Microarray Data User's Guide. 2010. Software manual available from http://bioconductor.org/packages/2.6/bioc/vignettes/limma/inst/ doc/usersguide.pdf Date last updated: April 3, 2010.
27 Bartels CL, Tsongalis GJ. MicroRNAs: novel biomarkers for human cancer. Clin Chem 2009; 55: 623-631.

28 Roush S, Slack FJ. The let-7 family of microRNAs. Trends Cell Biol 2008; 18: 505-516.

29 Takamizawa J, Konishi H, Yanagisawa K, et al. Reduced expression of the let-7 microRNAs in human lung cancers in association with shortened post-operative survival. Cancer Res 2004; 64: 3753-3756.

30 Yanaihara N, Caplen N, Bowman E, et al. Unique microRNA molecular profiles in lung cancer diagnosis and prognosis. Cancer Cell 2006; 9: 189-198.

31 Chang TC, Yu D, Lee YS, et al. Widespread microRNA repression by Myc contributes to tumourigenesis. Nat Genet 2008; 40: 43-50.

32 Urbich C, Kuehbacher A, Dimmeler S. Role of microRNAs in vascular diseases, inflammation, and angiogenesis. Cardiovasc Res 2008; 79: 581-588.

33 Landais S, Landry S, Legault $\mathrm{P}$, et al. Oncogenic potential of the miR-106-363 cluster and its implication in human T-cell leukemia. Cancer Res 2007; 67: 5699-5707.

34 Katada T, Ishiguro H, Kuwabara $\mathrm{Y}$, et al. microRNA expression profile in undifferentiated gastric cancer. Int J Oncol 2009; 34: 537-542.

$35 \mathrm{Wu}$ F, Zhu S, Ding Y, et al. MicroRNA-mediated regulation of Ubc9 expression in cancer cells. Clin Cancer Res 2009; 15: 1550-1557.

$36 \mathrm{Hu} \mathrm{Z}$, Chen $\mathrm{X}$, Zhao $\mathrm{Y}$, et al. Serum microRNA signatures identified in a genome-wide serum microRNA expression profiling predict survival of non-small-cell lung cancer. J Clin Oncol 2010; 28: 1721-1726. 